\title{
The Progenitor Systems and Explosion Mechanisms of Supernovae
}

\author{
Dan Milisavljevic* ${ }^{*}$ \\ Harvard University \\ E-mail: dmilisavecfa.harvard.edu
}

Supernovae are among the most powerful explosions in the universe. They affect the energy balance, global structure, and chemical make-up of galaxies, they produce neutron stars, black holes, and some gamma-ray bursts, and they have been used as cosmological yardsticks to detect the accelerating expansion of the universe. Fundamental properties of these cosmic engines, however, remain uncertain. In this review we discuss the progress made over the last two decades in understanding supernova progenitor systems and explosion mechanisms. We also comment on anticipated future directions of research and highlight alternative methods of investigation using young supernova remnants.

Frank N. Bash Symposium 2013: New Horizons in Astronomy

October 6-8, 2013

Austin, Texas

\footnotetext{
* Speaker.

${ }^{\dagger}$ Many thanks to R. Fesen, A. Soderberg, R. Margutti, J. Parrent, and L. Mason for helpful discussions and support during the preparation of this manuscript.
} 

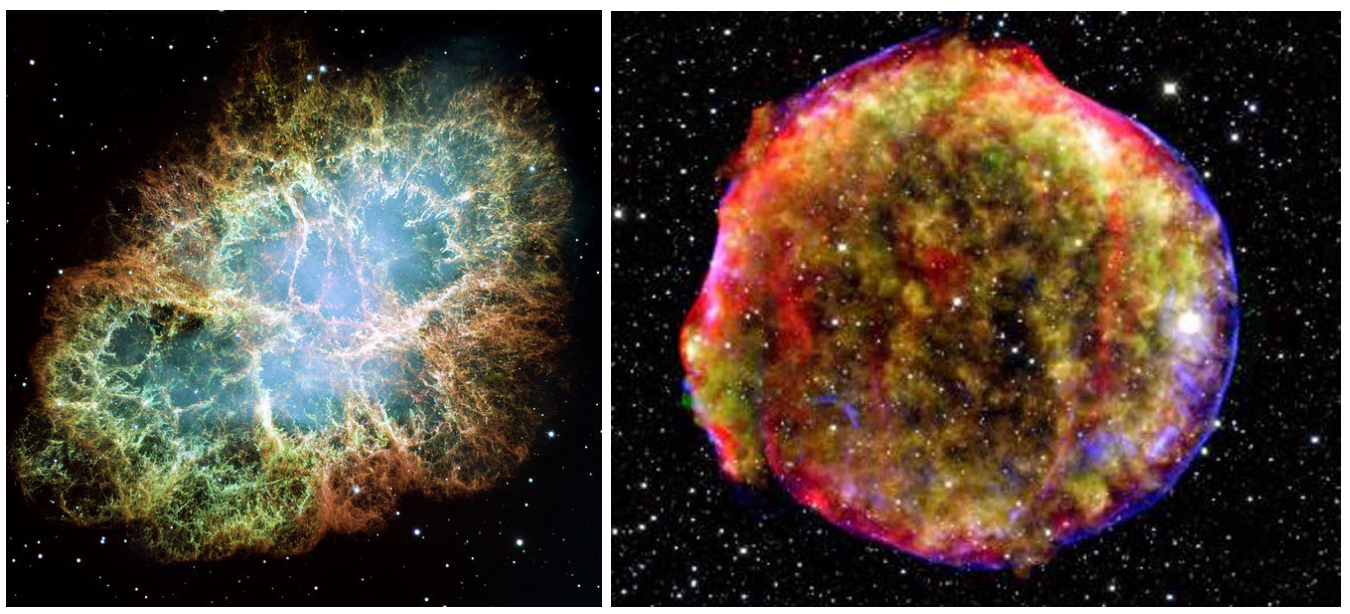

Figure 1: Left: Hubble Space Telescope image of the Crab Nebula as observed in the optical. This is the remnant of the original explosion of SN 1054. Credit: NASA/ESA/J.Hester/A.Loll. Right: Multiwavelength composite image of Tycho's supernova remnant. This is associated with the explosion of SN 1572. Credit NASA/CXC/SAO (X-ray); NASA/JPL-Caltech (Infrared); MPIA/Calar Alto/Krause et al. (Optical).

\section{Introduction}

Supernovae are the energetic explosions of stars. They affect the energy balance, global structure, and chemical make-up of the universe, help trigger star formation, are likely a major source of dust, produce neutron stars, pulsars, black holes, and some gamma-ray bursts, and seed the universe with heavy elements that make life possible. They are also used as standard candles in determining cosmological distances and played an important role in the discovery that the universe's expansion is accelerating.

However, several fundamental questions remain regarding the nature of supernovae. Simple questions such as "What stars explode?" and "How do stars explode?" do not have clear answers. Decades of investigation have shown that there are likely many channels to supernova explosions. In this short review we attempt to tie together developments related to supernova progenitor systems and explosion mechanisms made in the last two decades. We start with an introduction informed with historical context, then explore present understanding of the various types of supernovae, and end with anticipated topics of future investigation. Some emphasis will be placed on attempts to uncover powerful information about supernova progenitors and explosion dynamics from detailed observations of young supernova remnants.

\subsection{Historical Context}

The earliest recorded Galactic supernova was viewed by Chinese astronomers in $185 \mathrm{AD}$ and is now referred to as SN 185. The brightest ever recorded was SN 1006, which peaked at -7.5 in apparent visual magnitude. SN 1054 produced what we now observe as the Crab Nebula (Figure 1). Centuries passed before one of the most famous of Galactic supernovae was spotted in 1572. Tycho Brahe, only 25 at the time, made detailed observations of this event. What we see in this location today is now known as Tycho's supernova remnant and is thought to be the result of a Type Ia 

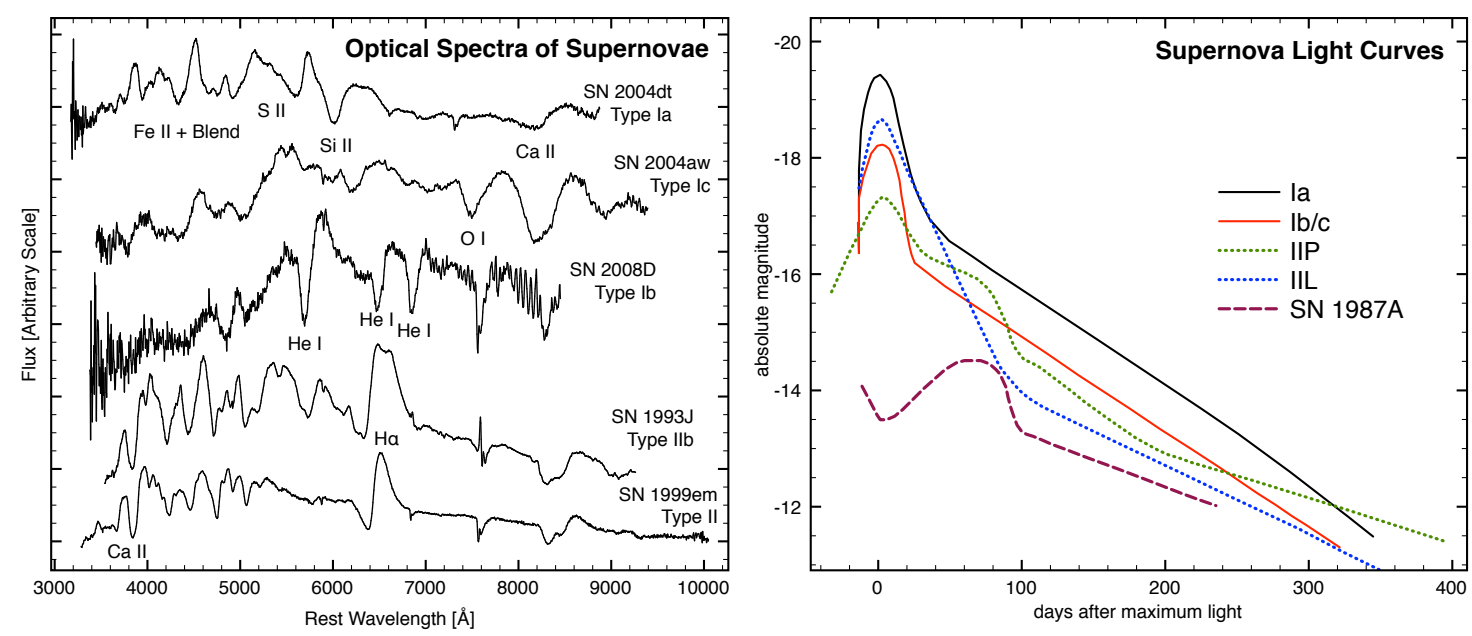

Figure 2: Left: Optical spectra of supernovae within weeks of maximum light. Data have been downloaded from the SUSPECT database at http://nhn.nhn.ou.edu/ suspect/ which came from the original sources [59]. Right: Representative light curves of supernovae during the first months after explosion. Adapted from [10].

supernova (Figure 1). Brahe's observations demonstrated that the "new star" did not move in its position for the months that it was visible. He argued that this was proof that the Aristotelian idea that the universe beyond the Moon and planets was immutable was wrong. Only 32 years later after Tycho's observations and three short years after his death, former assistant Johannes Kepler made detailed observations of the next recorded Galactic supernova SN 1604. This is believed to have been another Type Ia explosion and is observed today as Kepler's supernova remnant.

The next well-observed, well-studied, nearby event occurred in the Andromeda galaxy in 1885. It is referred to as SN 1885A or S Andromedae. SN 1885A reached a magnitude of 5.8. It occurred during the age of the telescope and was monitored closely. Spectroscopy was conducted but examined only visually and no photographic recordings remain. A century later, around the same time that the remnant of SN 1885A was discovered by [1] after decades of failed attempts, the closest supernova in the era of modern astronomy occurred: SN 1987A. It was discovered by Ian Shelton and Oscar Duhalde at Las Campanas Observatory in Chile on February 24, 1987 in the outskirts of the Tarantula Nebula in the Large Magellanic Cloud [2]. A burst of neutrinos were detected a few hours before visible light from the supernova reached Earth [3]. This confirmed predictions that neutrino emission associated with core collapse and formation of a neutron star preceded the sharp increase in visible light which occurs only after the shock wave breaks through the star's surface [4].

\subsection{Supernova Classification}

Modern classification of supernovae follows a spectroscopic system based on the lack or presence of spectral lines seen at optical wavelengths. The original system was proposed by [11]. The most basic division is between Type I and II events. Type I supernovae lack conspicuous features associated with hydrogen and Type II supernovae show clear hydrogen lines. Since Minkowski's 
time, the binary classification has branched considerably and is being continually updated in the face of new objects that bridge subtypes and extend luminosity ranges.

Recent discussions of supernova subtypes can be found in [10] and [12]. Type I are divided into three subclasses. Type Ia show Si II features, as well as strong sulfur lines, calcium lines, and a blend of lines associated with iron-peak elements including Fe II and Co II. Type Ib supernovae do not show strong Si II, but do show conspicuous lines of He I. The Type Ic class applies for objects showing weak or absent features of Si II and He I in their spectra. Type II show strong P-Cygni profiles of $\mathrm{H}$ Balmer lines. A transitional class known as Type IIb show Type II features at early times, but Type Ib features at late times. Example spectra of various supernovae obtained within weeks of optical maximum are showed in Figure 2.

Additional classification of supernovae can follow from the shape of its light curve. Type II supernova may be classified as Type IIP or IIL depending on whether they exhibit a "plateau" or "linear" decline in the months after explosion. In Figure 2 representative light curves are shown. The light curves of supernovae encode information about the size of the progenitor star, the mass of ejecta, its composition and density, and the supernova's explosion energy.

\section{Type Ia supernovae}

Type Ia supernovae are thought to be explosions of degenerate carbon-oxygen white dwarfs. A runaway thermonuclear explosion is initiated as a result of mass transfer in some type of close binary system [13-15]. They are observed in elliptical and spiral galaxies and thus associated with young and old stellar populations. Two progenitor explosion scenarios are most often cited: 1) the merger of two white dwarfs, referred to as a double degenerate (DD) explosion, or 2) a single white dwarf that gains mass from a companion main sequence, helium, or red giant star, referred to as a single degenerate (SD) explosion [16].

Some important papers include the following: [17] were among the first to explain the explosion of a Type I supernova using a white dwarf progenitor star; [18] provided the framework of understanding the light curves via radioactive decay of freshly synthesized ${ }^{56} \mathrm{Ni}$ in the cascade ${ }^{56} \mathrm{Ni} \rightarrow{ }^{56} \mathrm{Co} \rightarrow{ }^{56} \mathrm{Fe}$; [15] presented simulations of the explosion of a carbon white dwarf, and the W7 model from this paper is a standard one still in use today (e.g., [19]).

\subsection{Progenitor systems}

Uncertainty in the progenitor systems of Type Ia supernovae is due largely to the fact that they occur at large distances where it is impossible to detect stars prior to explosion. Thus, the discovery of SN $2011 \mathrm{fe}$ in M101 ( $D \sim 7 \mathrm{Mpc}$ ) - the closest Type Ia supernova in 25 years - was important as it provided one of the best opportunities to study the nature of Type Ia explosions [20]. Pre-explosion Hubble Space Telescope images of the region around the supernova did not reveal an obvious progenitor star system. Using these images [21] found that the luminosity of the progenitor system (especially the companion star) is 10-100 times fainter than previous limits on other Type Ia supernova progenitor systems. This deep limit allowed them to rule out luminous red giants and almost all helium stars as the mass-donating companion to the exploding white dwarf.

Approaching the issue of Type Ia supernova progenitor systems via a different route, [22] looked at Hubble Space Telescope images of the central region of the supernova remnant SNR 
0509-67.5 in the Large Magellanic Cloud. This remnant is believed to be the site of a Type Ia supernova that took place $400 \pm 50 \mathrm{yr}$ ago. They found that the central region contains no excompanion star to a visual magnitude limit of 26.9 (an absolute magnitude of $M_{V}=+8.4$ ). They claim that the lack of any ex-companion star to deep limits rules out all published single-degenerate models for this supernova and that the progenitor of this particular Type Ia supernova must have been a double-degenerate system. Objections to this interpretation, however, have been made (e.g., [23]).

Considerable and equally compelling evidence for SD degenerate scenarios exists. For example, [24] reported the spectroscopic detection of circumstellar material in a normal Type Ia supernova explosion. They found that the expansion velocities, densities, and dimensions of the circumstellar envelope to be consistent with the material having been ejected from the progenitor system. Relatively low expansion velocities of the circumstellar material suggest that the white dwarf was accreting material from a companion star that was in the red-giant phase at the time of the explosion.

Additional evidence for SD scenarios comes from examples of Type Ia supernovae that interact with H-rich circumstellar material. SN 2002ic [25] and SN 2005gj [26] are well-known examples of this type of interaction. PTF11 kx is another example that was observed shortly after outburst and exhibited the interaction strongly [27]. Using early-time data [27] concluded that PTF11kx was a bona fide Type Ia supernova with a symbiotic nova progenitor. Further evidence for this interpretation came from late-time data presented in [28].

\subsection{Explosion mechanisms}

The nature of Type Ia explosions remains poorly understood. Scenarios of explosions proceeding via detonation (a super-sonic shock wave) or deflagration (a subsonic burning wave) have been modeled with only partial success. The problem is that thermonuclear runaway models involving a pure detonation or a deflagration do not yield light curves that agree with photometric observations or the correct distribution of Fe-peak and intermediate mass elemental abundances that match the observed spectral observations.

A pure detonation explosion of a white dwarf proceeds faster than the white dwarf's internal sound speed. Thus, the star's outer layers do not have time to expand or react to the outgoing burning shock wave and the swift burning nearly completely converts the star into iron group elements, especially ${ }^{56} \mathrm{Ni}$. Alternatively, in a deflagration explosion the star does have time to expand. Burning occurs in lower density material and leads to less production of ${ }^{56} \mathrm{Ni}$ and the synthesis of more intermediate mass elements.

The best solution thus far has been to incorporate both explosion processes. One of the first proponents of this scenario was [29]. Transition from deflagration to detonation allows the star time to expand (during the initial deflagration phase) resulting in lower densities in the outer parts of the white dwarf. This, in turn, leads to less complete burning of the outer layers into ${ }^{56} \mathrm{Ni}$ and intermediate mass element abundances in line with early time spectral observations.

SN 2011fe was discovered within 11 hours of explosion and was monitored closely thereafter [20]. These exhaustive, high-cadence observations of SN 2011fe were used by [30] to explore whether they could be used to constrain Type Ia supernova explosion scenarios using advanced three-dimensional simulations of (i) a delayed detonation in a Chandrasekhar-mass white dwarf 
and (ii) a violent merger of two white dwarfs. A clear preference for one model over the other was not found (see also [19] and [31]).

\section{Type Ib, Ic, and II - Core-collapse Supernovae}

Type Ib, Ic, and II supernovae occur exclusively in spiral galaxies and are generally associated with the core-collapses of massive stars $\left(\geq 8 \mathrm{M}_{\odot}\right)$. They succumb to this violent death when nuclear fusion suddenly becomes unable to sustain the core against its own gravity and are a primary source of heavy elements in the universe.

\subsection{Progenitor systems}

It is thought that the spectral classifications of Type II, IIb, Ib, and Ic may follow an order consistent with the progenitor stars having been increasingly stripped of their outer $\mathrm{H}$ - and He-rich envelopes [32]. There have been unexpected discoveries with core-collapse progenitor stars. For instance, it came as something of a surprise that the progenitor star of SN 1987A was the blue supergiant Sanduleak $-69^{\circ} 202$ because it went against the theoretical expectation that Type II supernovae come from red supergiants.

Real progress has been made in the last twenty years with regard to the progenitor systems of core-collapse supernovae thanks in large part to high resolution images of the Hubble Space Telescope. Pre-explosion images of progenitor systems has established fairly well that at least some Type II events do come from red supergiants [33]. A handful of progenitors of Type IIb have also been imaged. The first, before the Hubble Space Telescope era, was SN 1993J, which was associated with a K0I star [35]. Most recently, high-resolution pre- and post-explosion images of the region around the Type IIb SN 2011dh demonstrated that its progenitor was a yellow supergiant [36].

The progenitor stars of Type Ib and Ic supernovae have been difficult to find. They have been theorized to be Wolf Rayet stars since they can be largely devoid of H-rich envelopes [37]. There have been possible detections of progenitor stars of of Type Ib supernovae; iPTF13bvn is a recent example [38]. However, no detections so far have been conclusive. This lack of detection has been used to argue that these supernovae should originate from moderate mass interacting binaries.

Many supernovae show evidence for episodic mass loss prior to explosion. An extreme case is SN 2009ip, which was a supernova that interacted with an extensive circumstellar environment [39]. ${ }^{1}$ However, it may be that less extreme mass loss episodes occur in a significant fraction of progenitor systems. As seen in Figure 3, radio light curve variations are observed in a variety of supernovae including SN 2001ig [40], SN 2008ax [41], and SN 2003bg [42]. These fluctuations are similar in both timescale and amplitude and have been reasonably explained in terms of density modulations in the pre-explosion environment shaped by the progenitor system. [43] note that it is intriguing how the radio modulations have also been observed in relativistic, engine-driven $\mathrm{SNe}$ including the gamma-ray burst-associated SN 1998bw [44, 45] and SN 2009bb [46]. The flux density modulations in these cases were attributed to energy injection from the central engine, but the

\footnotetext{
${ }^{1}$ SN 2009ip was a Type IIn supernova. Type IIn supernovae are associated with narrow spectral features (full width at half maximum $<200 \mathrm{~km} \mathrm{~s}^{-1}$ ) and are not reviewed here.
} 


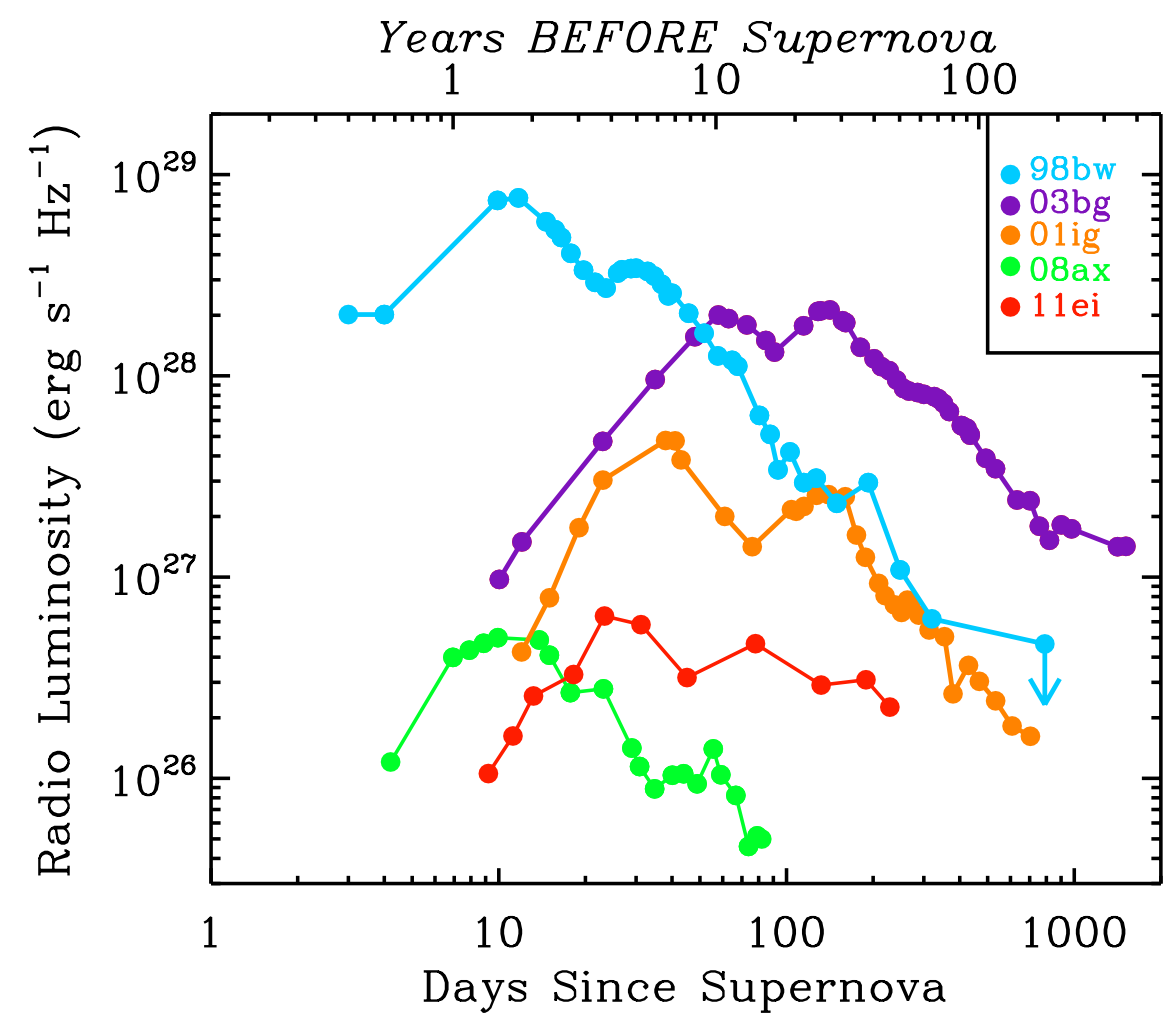

Figure 3: The $5 \mathrm{GHz}$ light curve for various supernovae. Bottom $x$-axis represents days since supernova outburst and the top $x$-axis represents inferred times of circumstellar material density modulations in years previous to outburst (assuming $v_{w}=1000 \mathrm{~km} \mathrm{~s}^{-1}$ ). All exhibit variable radio emission that deviates from the expected decay as roughly $F_{V} \propto t^{-1}$. From [43].

resemblance to radio modulations of non-relativistic supernovae suggest that density fluctuations in circumstellar material on radial scales of $\lesssim 10^{17} \mathrm{~cm}$ may be common among stripped-envelope supernova explosions.

\subsection{Explosion mechanisms}

It was [47] who first proposed that supernovae are energized by the collapse of an ordinary star to a neutron star. Since then, hundreds of studies have attempted to understand how such a process can work in detail; pioneering papers include [48] and [49]. The process is thought to start with a massive star $>8 \mathrm{M}_{\odot}$ that has undergone successive stages of hydrogen, helium, carbon, neon, oxygen, and silicon fusion at its core. Eventually, a core of Fe-group elements is produced with a total mass that exceeds the Chandrasekhar limit $\left(\sim 1.5 \mathrm{M}_{\odot}\right)$. Nuclear binding energy per nucleon reaches maximum at the Fe-group, so no further energy can be released by nuclear fusion. The high temperatures and densities induce electron capture by nuclei and photodisintegration, the loss of electrons robs the core of crucial pressure, and the iron core collapses.

The explosion that is predicted to follow is not well understood. Collapse halts when the repulsive component of the nuclear force kicks in at densities around $4-5 \times 10^{14} \mathrm{~g} \mathrm{~cm}^{-3}$. A rebound generates a shock wave as the outer half of the core continues to crash down. It was long- 

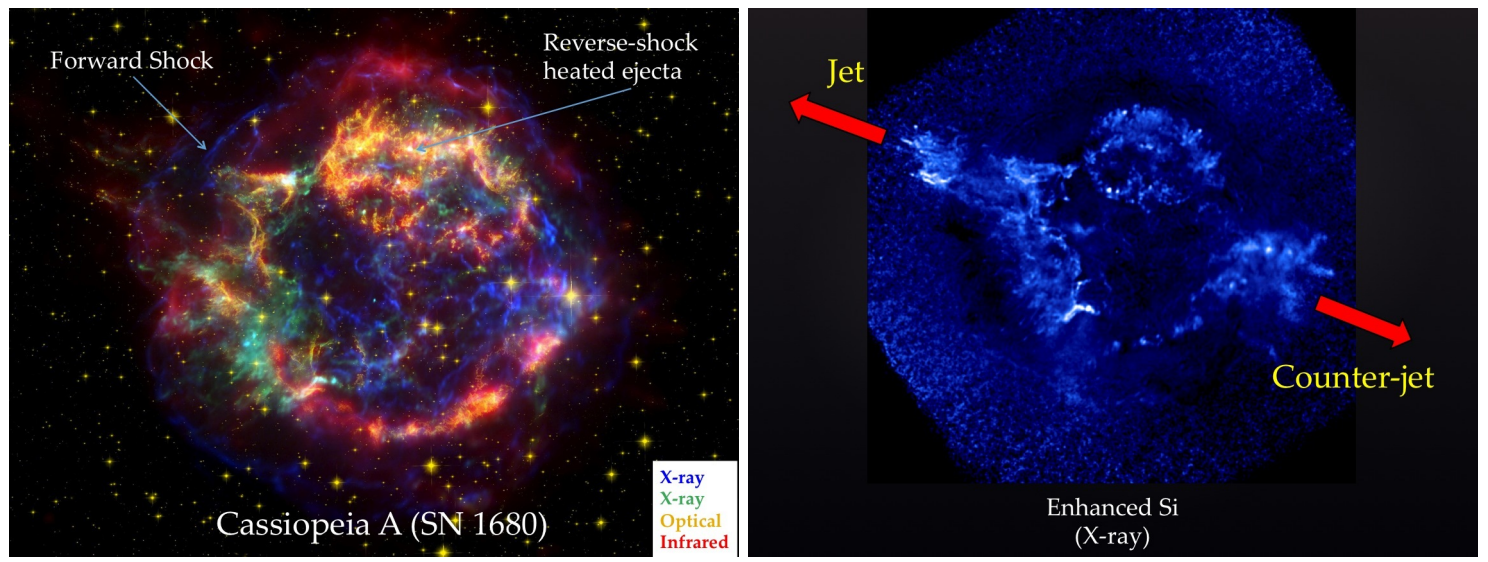

Figure 4: Left: Multi-wavelength composite image of the O-rich, Galactic supernova remnant Cassiopeia A. Optical data obtained with the Hubble Space Telescope, near-infrared data with the Spitzer Space Telescope, and X-ray with the Chandra X-ray Observatory. Credit: NASA/JPL-Caltech. Right: Enhanced Si image of Cassiopeia A made from X-ray data. Credit: NASA/CXC/GSFC/U.Hwang et al. The outflows (or 'jets') of high-velocity material in the NE and SW regions are labeled.

believed that this bounce-shock mechanism might be the driver of supernova explosions. However, modern simulations have demonstrated that (at least for stars outside the $8-10 \mathrm{M}_{\odot}$ range) the outgoing shock is not energetic enough [50-53]. The shock wave stalls because of continued photodisintegration and copious neutrino losses. A few milliseconds after the bounce the outward motion stops and the dense, hot neutron-rich core begins to rapidly accrete in-falling mass.

Many studies have explored a variety of possible mechanisms by which the stalled shock can be re-invigorated. Aspericity in the explosion appears to be of crucial importance. The origin of expansion asphericities is currently uncertain. Possible causes include asymmetric neutrino heating and accretion-shock instabilities [54-59], and the influences of rotation and magnetic fields [6064].

Observations of the kinematic and chemical properties of supernova ejecta can help investigate which of the aforementioned explosion mechanisms may dominate. For example, late-time optical spectra obtained $t>6$ months beyond outburst in stripped-envelope events often exhibit multipeaked emission line profiles consistent with aspherical axisymmetric and potentially jet-related explosions viewed along different angles from the equatorial plane [65-69]. Additional clues for constraining explosion mechanisms come from spectropolarimetry. These studies show that ejecta can be asymmetric in the inner layers, supporting the view that the explosion process is strongly aspherical [70, 71].

\section{Young Supernova Remnants}

An alternative way to investigate core-collapse supernova explosions is through observations of young supernova remnants. Studies of young O-rich Galactic remnants allow one to probe kinematic asymmetries in the expanding ejecta at spatial and kinematic scales not possible from extragalactic observations. They can also offer clues about the explosive mixing of chemically distinct zones in the progenitor star and the nature of the central compact remnant. 

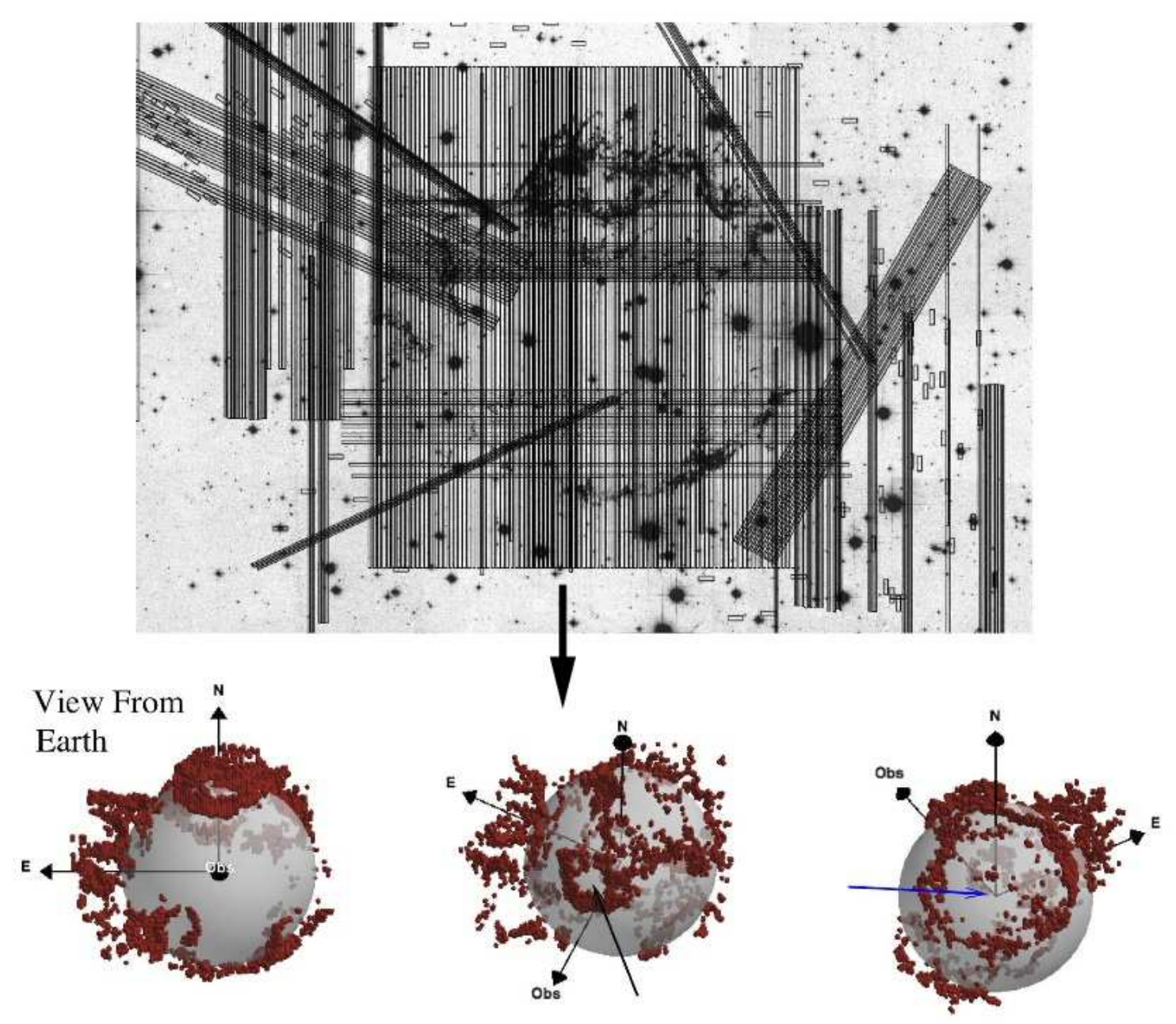

Figure 5: Data from [80] showing the kinematic map constructed for Cas A. Top: Locations of over 200 slit positions used to develop a 3D reconstruction. Bottom: Various perspectives of the resulting 3D map. Blue arrow points to a region coincident with X-ray emitting Fe-rich material.

\subsection{Cassiopeia A}

The young Galactic remnant Cassiopeia A (Cas A) provides perhaps the clearest look at the explosion dynamics of a high mass supernova (Figure 4). With an explosion date most likely around 1680, Cas A is the youngest Galactic core-collapse supernova remnant known [72]. At an estimated distance of $3.4 \mathrm{kpc}$ [73], it is also among the closest. Cas A is inferred to have undergone extensive mass loss from its original $20-25 \mathrm{M}_{\odot}$ progenitor to only $3-4 \mathrm{M}_{\odot}$ upon explosion, leaving behind a relatively dense and slow moving remnant stellar wind [74]. This may have required the existence of a binary companion to aid the mass loss [75].

Cas A is the only historical core-collapse supernova remnant with a secure supernova subtype classification. The detection of light echoes of the supernova outburst [76-78] enabled follow-up optical spectral observations that determined the original supernova to have exhibited an optical spectrum at maximum light similar to those seen for the Type IIb events SN 1993J and SN 2003bg $[79,77]$. 


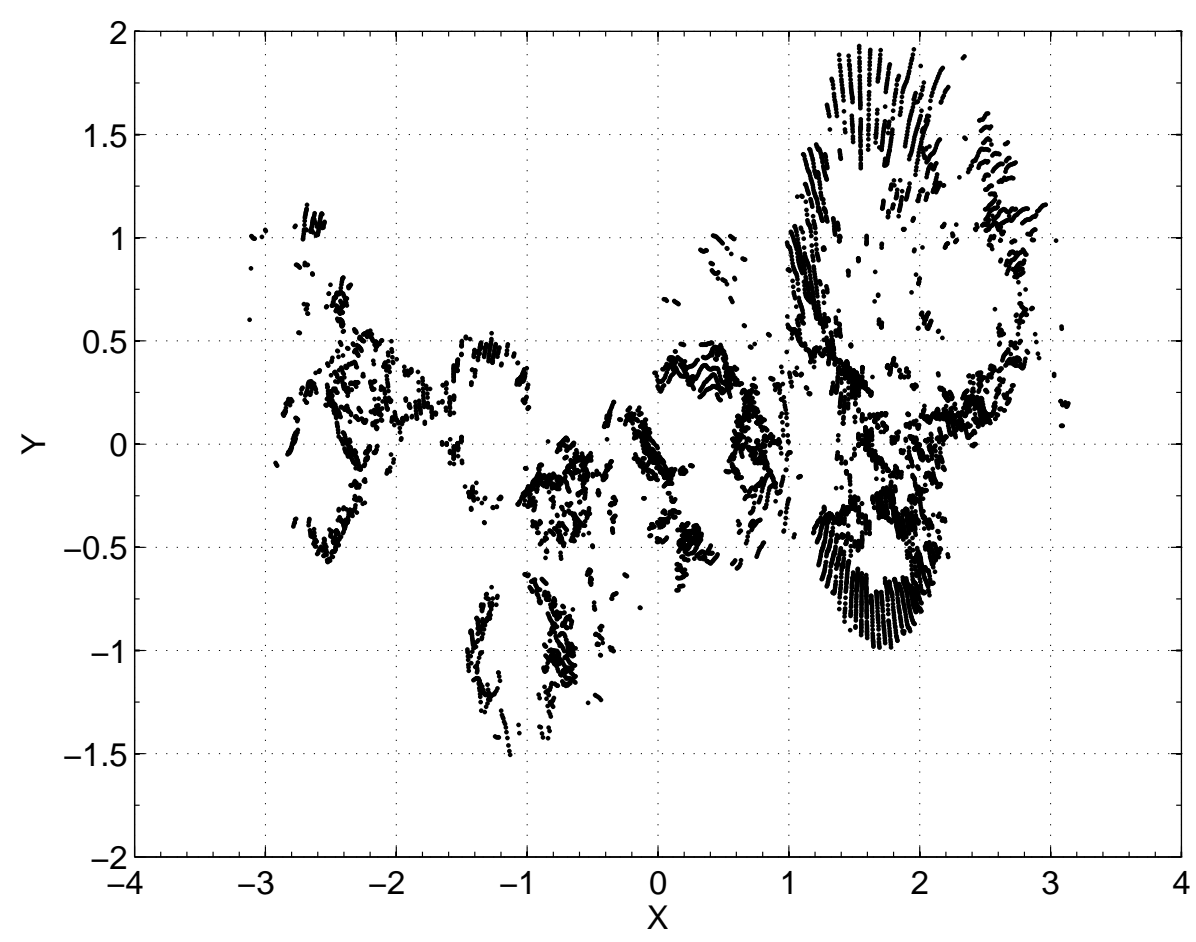

Figure 6: The main shell of Cas A's optically-emitting ejecta as represented in a Mercator projection. From [80].

[80] recently created a detailed 3D kinematic reconstruction of Cas A (Figure 5). Its opticalemitting ejecta were mapped from a spectroscopic survey involving hundreds of long slit spectra taken over several years and represents the most complete kinematic map of a supernova remnant in the optical to date. The reconstruction shows that Cas A's main shell ejecta are arranged in several well-defined and nearly circular rings with diameters between approximately $30^{\prime \prime}(0.5 \mathrm{pc})$ and $2^{\prime}$ (2 pc). In Figure 6, a Mercator projection of the main shell knots is shown to illustrate the relative scale and distribution of the rings. Some rings form complete circles, while others appear as partial circles or ellipses.

Motivation to undertake a deep reconnaissance of the entire Cas A was driven in part from an interest in finally grasping the kinematic properties of the NE and SW regions of exceptionally high velocity $\left(1-1.5 \times 10^{4} \mathrm{~km} \mathrm{~s}^{-1}\right)$ material. Previous studies could not address the question of whether they form a true bipolar structure. The survey demonstrated that the outflows appear to be directed in nearly opposite directions with opening half-angles of approximately 40 degrees. This makes the outflows inconsistent with a highly-collimated (opening half-angle $<10$ degrees) jet-induced explosion (e.g., [81, 60, 61]), but still puzzling because their chemistry is consistent with an origin from the Si-S-Ca-Ar layer deep within the progenitor star.

The important lesson from Cas A is that the distribution of its metal-rich ejecta is not random. The sizes and arrangement of the large-scale rings may be informing us about important properties of the explosion dynamics and subsequent evolution of the expanding debris. The distribution of iron - which is a good tracer of the explosion dynamics - with respect to the main shell ejecta is intriguing. In Figure 7 the optically-emitting S-rich and O-rich main shell ejecta are shown along 

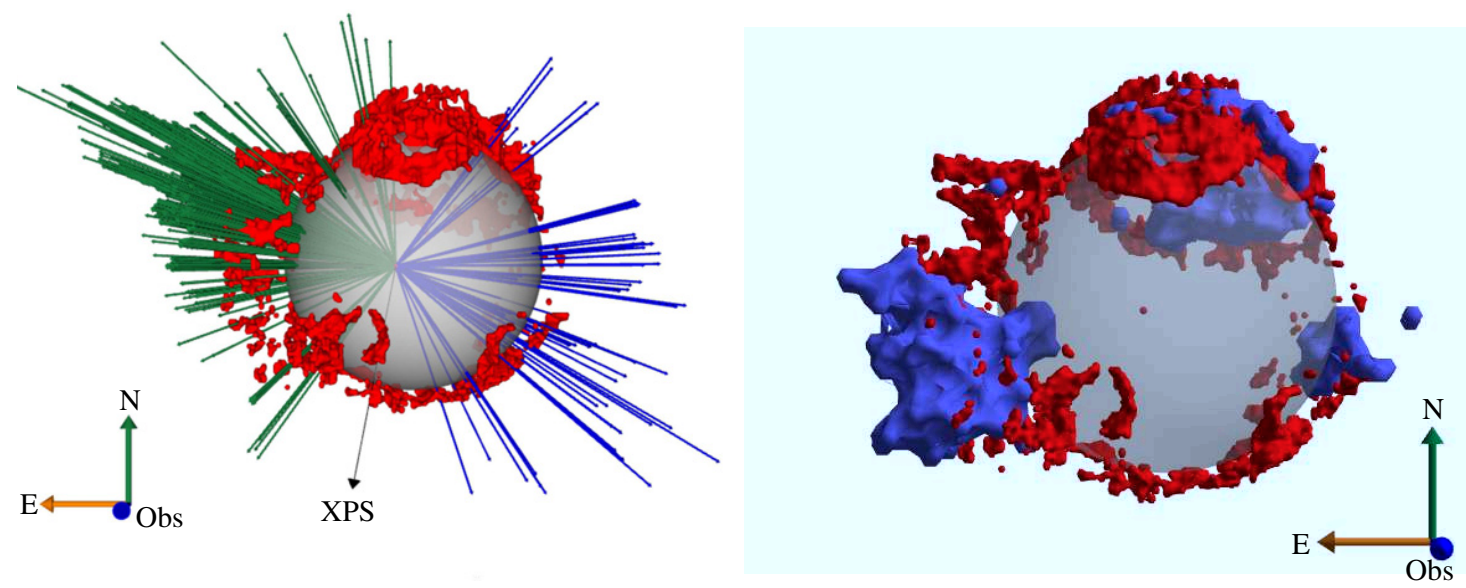

Figure 7: Left: Vector plots of the high velocity ejecta knots (colored green and blue, respectively) shown along with the main shell (colored red). Right: Location of iron-rich X-ray emitting ejecta (blue) with respect to the main shell sulfur- and oxygen-rich ejecta. Optical data are from [80] and X-ray data are from [82].

with the location of X-ray emitting iron-rich material measured from Chandra data presented in [82]. Interestingly, each of the three large concentrations of Fe-rich ejecta lie within and bounded by ring structures. This complementary arrangement is consistent with a causal relationship.

The ejecta rings are not unlike large-scale features seen in supernova explosion models (e.g., [83]) and may have origin in part to a "Ni bubble effect" having influenced the remnant's expansion dynamics shortly after the original explosion. Indeed, it's possible that the observed ejecta rings represent cross-sections of large cavities in the expanding ejecta created by a post-explosion input of energy from plumes of radioactive ${ }^{56} \mathrm{Ni}$-rich ejecta [84].

\subsection{Broader Implications}

Recent 3D reconstructions of other young core-collapse supernova remnants have suggested that many of the kinematic properties observed in Cas A are not unique. Although only a handful of known supernova remnants are appropriate for this kind of analysis, those that have been studied have revealed evidence of the same large-scale ejecta rings, velocity asymmetries, and high-velocity bipolar asymmetries as observed in Cas A. Thus, information learned from Cas A may have implications for a variety of supernovae and supernova remnants.

Indeed, direct connections between Cas A and extragalactic supernovae have been made. [85] summed all main shell spectra into a single, integrated spectrum, mimicking what the remnant would appear as as an unresolved extragalactic source. Intriguingly, close similarities were found between the integrated Cas A spectrum and several late-time optical spectra of decades-old extragalactic supernovae, including SN 1979C, SN 1993J, SN 1980K, and the ultra-luminous supernova remnant in NGC 4449. These spectra all showed pronounced blueshifted emission with conspicuous line substructure in oxygen, sulfur, and argon. Because the emission line substructure observed in Cas A is associated with large-scale rings of ejecta, it is possible that the similar line substructure observed in intermediate-aged supernovae are also associated with large-scale rings of ejecta. 


\section{Conclusions}

Fundamental questions remain about the nature of supernova progenitor systems and explosion mechanisms. With regard to Type Ia explosions, the pendulum swings back and forth between single-degenerate and double-degenerate progenitor systems [86-88]. The observed inhomogeneity of these events has led to the growing speculation that two or more channels all contribute. The detonation-to-deflagration model is currently the best at reproducing empirical results, but cannot do so for all observables and the physical motivations behind this scenario are not well understood.

Real progress has been made with the progenitor systems of core-collapse supernovae. Direct imaging of supernova progenitor systems has demonstrated convincingly that Type II supernovae can come from red supergiant stars. The progenitors of stripped-envelope supernovae of Type IIb, $\mathrm{Ib}$, and Ic have been more difficult to understand. Detailed understanding of core-collapse explosion mechanisms may await the next generation of sophisticated 3D simulations. Investigations of young, nearby supernova remnants can provide unique information about the explosion dynamics and local environments of supernovae that will provide constraints for these simulations.

Looking further into the future, new facilities promise to revolutionize the field of transient astronomy. The Large Synoptic Survey Telescope will image the entire available night sky every few nights with a single visit depth of $r \sim 24.5$ mag to find $\sim 10^{5}$ supernova per year, and Advanced LIGO, which is sensitive to gravitational waves that carry live dynamical information (mass, angular momentum, nuclear equation of state) from deep inside the supernova core, will provide a novel probe of the core-collapse supernova explosion mechanism. These and other efforts of the upcoming decade will explore uncharted frontiers of high energy, extreme astrophysics and (with a little luck) will shed new light on supernovae.

\section{References}

[1] Fesen, R. A., Saken, J. M., \& Hamilton, A. J. S. 1989, Astrophysical Journal Letters, 341, L55

[2] Kunkel, W., Madore, B., Shelton, I., et al. 1987, IAU Circular, 4316, 1

[3] Hirata, K., Kajita, T., Koshiba, M., Nakahata, M., \& Oyama, Y. 1987, Physical Review Letters, 58, 1490

[4] Burrows, A., \& Lattimer, J. M. 1986, Astrophysical Journal, 307, 178

[5] Altavilla, G., Stehle, M., Ruiz-Lapuente, P., et al. 2007, Astronomy and Astrophysics, 475, 585

[6] Taubenberger, S., Pastorello, A., Mazzali, P. A., et al. 2006, Monthly Notices of the Royal Astronomical Society, 371, 1459

[7] Modjaz, M., Li, W., Butler, N., et al. 2009, Astrophysical Journal, 702, 226

[8] Matheson, T., Filippenko, A. V., Barth, A. J., et al. 2000, Astronomical Journal, 120, 1487

[9] Hamuy, M., Pinto, P. A., Maza, J., et al. 2001, Astrophysical Journal, 558, 615

[10] Filippenko, A. V. 1997, Annual Review of Astronomy and Astrophysics, 35, 309

[11] Minkowski, R. 1941, Proceedings of the Astronomical Society of the Pacific, 53, 224

[12] Turatto, M., Benetti, S., \& Cappellaro, E. 2003, From Twilight to Highlight: The Physics of Supernovae, 200 
[13] Hoyle, F., \& Fowler, W. A. 1960, Astrophysical Journal, 132, 565

[14] Colgate, S. A., \& McKee, C. 1969, Astrophysical Journal, 157, 623

[15] Nomoto, K., Thielemann, F.-K., \& Yokoi, K. 1984, Astrophysical Journal, 286, 644

[16] Hillebrandt, W., \& Niemeyer, J. C. 2000, Annual Review of Astronomy and Astrophysics, 38, 191

[17] Hansen, C. J., \& Wheeler, J. C. 1969, Astrophysics and Space Science, 3, 464

[18] Arnett, W. D. 1979, Astrophysical Journal Letters, 230, L37

[19] Mazzali, P., Sullivan, M., Hachinger, S., et al. 2013, arXiv:1305.2356

[20] Nugent, P. E., Sullivan, M., Cenko, S. B., et al. 2011, Nature, 480, 344

[21] Li, W., Bloom, J. S., Podsiadlowski, P., et al. 2011, Nature, 480, 348

[22] Schaefer, B. E., \& Pagnotta, A. 2012, Nature, 481, 164

[23] Meng, X., \& Podsiadlowski, P. 2013, Astrophysical Journal Letters, 778, L35

[24] Patat, F., Chandra, P., Chevalier, R., et al. 2007, Science, 317, 924

[25] Wang, L., Baade, D., Höflich, P., et al. 2004, Astrophysical Journal Letters, 604, L53

[26] Aldering, G., Antilogus, P., Bailey, S., et al. 2006, Astrophysical Journal, 650, 510

[27] Dilday, B., Howell, D. A., Cenko, S. B., et al. 2012, Science, 337, 942

[28] Silverman, J. M., Nugent, P. E., Gal-Yam, A., et al. 2013, Astrophysical Journal, 772, 125

[29] Khokhlov, A. M. 1991, Astronomy and Astrophysics, 245, 114

[30] Röpke, F. K., Kromer, M., Seitenzahl, I. R., et al. 2012, Astrophysical Journal Letters, 750, L19

[31] Dessart, L., Blondin, S., Hillier, D. J., \& Khokhlov, A. 2013, arXiv:1310.7747

[32] Nomoto, K. I., Iwamoto, K., \& Suzuki, T. 1995, Physics Reports, 256, 173

[33] Smartt, S. J. 2009, Annual Review of Astronomy and Astrophysics, 47, 63

[34] Clocchiatti, A., Wheeler, J. C., Phillips, M. M., et al. 1997, Astrophysical Journal, 483, 6759

[35] Aldering, G., Humphreys, R. M., \& Richmond, M. 1994, Astronomical Journal, 107, 662

[36] Van Dyk, S. D., Zheng, W., Clubb, K. I., et al. 2013, Astrophysical Journal Letters, 772, L32

[37] Gaskell, C. M., Cappellaro, E., Dinerstein, H. L., et al. 1986, Astrophysical Journal Letters, 306, L77

[38] Cao, Y., Kasliwal, M. M., Arcavi, I., et al. 2013, Astrophysical Journal Letters, 775, L7

[39] Margutti, R., Milisavljevic, D., Soderberg, A. M., et al. 2014, Astrophysical Journal, 780, 21

[40] Ryder, S. D., Sadler, E. M., Subrahmanyan, R., et al. 2004, Monthly Notices of the Astronomical Society, 349, 1093

[41] Roming, P. W. A., Pritchard, T. A., Brown, P. J., et al. 2009, Astrophysical Journal Letters, 704, L118

[42] Soderberg, A. M., Chevalier, R. A., Kulkarni, S. R., \& Frail, D. A. 2006, Astrophysical Journal, 651, 1005

[43] Milisavljevic, D., Margutti, R., Soderberg, A. M., et al. 2013, Astrophysical Journal, 767, 71

[44] Kulkarni, S. R., Frail, D. A., Wieringa, M. H., et al. 1998, Nature, 395, 663 
[45] Li, Z.-Y., \& Chevalier, R. A. 1999, Astrophysical Journal, 526, 716

[46] Bietenholz, M. F., Soderberg, A. M., Bartel, N., et al. 2010, Astrophysical Journal, 725, 4

[47] Baade, W., \& Zwicky, F. 1934, Proceedings of the National Academy of Science, 20, 254

[48] Colgate, S. A., \& White, R. H. 1966, Astrophysical Journal, 143, 626

[49] Bethe, H. A., \& Wilson, J. R. 1985, Astrophysical Journal, 295, 14

[50] Mayle, R., \& Wilson, J. R. 1988, Astrophysical Journal, 334, 909

[51] Mezzacappa, A., Liebendörfer, M., Messer, O. E., et al. 2001, Physical Review Letters, 86, 1935

[52] Kitaura, F. S., Janka, H.-T., \& Hillebrandt, W. 2006, Astronomy and Astrophysics, 450, 345

[53] Janka, H.-T., Langanke, K., Marek, A., Martinez-Pinedo, G., Mueller, B. 2007, Physics Reports, 442, 38

[54] Kifonidis, K., Plewa, T., Janka, H.-T., Mueller, E. 2000, Astrophysical Journal Letters, 531, L123

[55] Blondin, J. M., Mezzacappa, A., \& DeMarino, C. 2003, Astrophysical Journal, 584, 971

[56] Kifonidis, K., Plewa, T., Janka, H.-T., Mueller, E. 2003, Astronomy and Astrophysics, 408, 621

[57] Burrows, A., Livne, E., Dessart, L., Ott, C. D., \& Murphy, J. 2006, Astrophysical Journal, 640, 878

[58] Scheck, L., Kifonidis, K., Janka, H.-T., Mueller, E. 2006, Astronomy and Astrophysics, 457, 963

[59] Hanke, F., Marek, A., Müller, B., \& Janka, H.-T. 2012, Astrophysical Journal, 755, 138

[60] Wheeler, J. C., Meier, D. L., \& Wilson, J. R. 2002, Astrophysical Journal, 568, 807

[61] Akiyama, S., Wheeler, J. C., Meier, D. L., \& Lichtenstadt, I. 2003, Astrophysical Journal, 584, 954

[62] Fryer, C. L., \& Warren, M. S. 2004, Astrophysical Journal, 601, 391

[63] Shibata, M., Liu, Y. T., Shapiro, S. L., \& Stephens, B. C. 2006, Physical Review D, 74, 104026

[64] Masada, Y., Takiwaki, T., Kotake, K., \& Sano, T. 2012, Astrophysical Journal, 759, 110

[65] Mazzali, P. A., Kawabata, K. S., Maeda, K., et al. 2005, Science, 308, 1284

[66] Modjaz, M., Kirshner, R. P., Blondin, S., Challis, P., \& Matheson, T. 2008, Astrophysical Journal Letters, 687, L9

[67] Maeda, K., Kawabata, K., Mazzali, P. A., et al. 2008, Science, 319, 1220

[68] Taubenberger, S., Valenti, S., Benetti, S., et al. 2009, Monthly Notices of the Royal Astronomical Society, 397, 677

[69] Milisavljevic, D., Fesen, R. A., Gerardy, C. L., Kirshner, R. P., \& Challis, P. 2010, Astrophysical Journal, 709, 1343

[70] Wang, L., \& Wheeler, J. C. 2008, Annual Review of Astronomy and Astrophysics, 46, 433

[71] Tanaka, M., Kawabata, K. S., Hattori, T., et al. 2012, Astrophysical Journal, 754, 63

[72] Thorstensen, J. R., Fesen, R. A., \& van den Bergh, S. 2001, Astronomical Journal, 122, 297

[73] Reed, J. E., Hester, J. J., Fabian, A. C., \& Winkler, P. F. 1995, Astrophysical Journal, 440, 706

[74] Chevalier, R. A., \& Oishi, J. 2003, Astrophysical Journal Letters, 593, L23

[75] Young, P. A., Fryer, C. L., Hungerford, A., et al. 2006, Astrophysical Journal, 640, 891 
[76] Rest, A., Welch, D. L., Suntzeff, N. B., et al. 2008, Astrophysical Journal Letters, 681, L81

[77] Rest, A., Foley, R. J., Sinnott, B., et al. 2011, Astrophysical Journal Letters, 732, 3

[78] Besel, M.-A., \& Krause, O. 2012, Astronomy and Astrophysics, 541, L3

[79] Krause, O., Birkmann, S. M., Usuda, T., et al. 2008, Science, 320, 1195

[80] Milisavljevic, D., \& Fesen, R. A. 2013, Astrophysical Journal, 772, 134

[81] Khokhlov, A. M., Höflich, P. A., Oran, E. S., et al. 1999, Astrophysical Journal Letters, 524, L107

[82] DeLaney, T., Rudnick, L., Stage, M. D., et al. 2010, Astrophysical Journal, 725, 2038

[83] Hammer, N. J., Janka, H.-T., Mueller, E. 2010, Astrophysical Journal, 714, 1371

[84] Blondin, J. M., Borkowski, K. J., \& Reynolds, S. P. 2001, Astrophysical Journal, 557, 782

[85] Milisavljevic, D., Fesen, R. A., Chevalier, R. A., et al. 2012, Astrophysical Journal, 751, 25

[86] Hachisu, I., Kato, M., \& Nomoto, K. 2012, Astrophysical Journal Letters, 756, L4

[87] Nomoto, K., Kamiya, Y., \& Nakasato, N. 2013, IAU Symposium, 281, 253

[88] Maoz, D., Mannucci, F., \& Nelemans, G. 2013, arXiv:1312.0628 